AperTO - Archivio Istituzionale Open Access dell'Università di Torino

\title{
A multiplex chemiluminescent biosensor for type B-fumonisins and aflatoxin B1 quantitative detection in maize flour
}

\section{This is the author's manuscript}

Original Citation:

Availability:

This version is available http://hdl.handle.net/2318/154078

since 2020-03-16T17:38:42Z

Published version:

DOI:10.1039/c4an01613k

Terms of use:

Open Access

Anyone can freely access the full text of works made available as "Open Access". Works made available under a Creative Commons license can be used according to the terms and conditions of said license. Use of all other works requires consent of the right holder (author or publisher) if not exempted from copyright protection by the applicable law. 


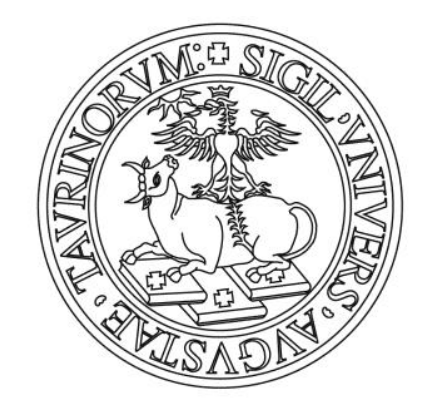

\section{UNIVERSITÀ DEGLI STUDI DI TORINO}

\section{This is an author version of the contribution published on:}

Questa è la versione dell'autore dell'opera:

M.Zangheri, F.Di Nardo, L.Anfossi, C.Giovannoli, C.Baggiani, A.Roda, M.Mirasoli

"Multiplex chemiluminescent biosensor for type B-fumonisins andaflatoxin B1 quantitative detection in maize flour" Analyst, 2015, 140, 358-65 (DOI

10.1039/C4AN01613K)

The definitive version is available at:

La versione definitiva è disponibile alla URL:

http://pubs.rsc.org/en/journals/journalissues/an\#!recentarticles\&all 


\title{
Multiplex chemiluminescent biosensor for type B-fumonisins and aflatoxin B1 quantitative detection in maize flour
}

\author{
Martina Zangheri, a Fabio Di Nardo, b Laura Anfossi, b Cristina Giovannoli, b Claudio \\ Baggiani, b Aldo Roda, a,c and Mara Mirasoli, *a,c
}

\section{Received 1st September 2014, Accepted 5 21st October 2014 DOI: 10.1039/C4AN01613K}

A multiplex chemiluminescent biosensor for simple, rapid and ultrasensititive on-site quantification of Aflatoxin B1 and type B-Fumonisins in maize samples has been developed. The biosensor integrates a multiplex indirect competitive lateral flow immunoassay (LFIA) based on enzyme-catalyzed 10 chemiluminescence detection and a highly sensitive portable charge-coupled device (CCD) camera, employed in a lensless "contact" imaging configuration. The developed assay requires a simple extraction of the analytes from maize flour samples followed by their detection with a 30-min assay time. The use of chemiluminescence detection allowed accurate and objective analytes quantification, enabling simultaneous detection of type B-Fumonisins and Aflatoxin B1 down to 6 /g kg-1 and $1.5 \int \mathrm{g}$ $\mathrm{kg}-1,15$ respectively, thus fulfilling the standards imposed by the legislation of European Union. Maize flour samples spiked with both analytes were subjected to multiplex analysis obtaining recoveries ranging from 79 to $119 \%$ and coefficient of variation below 20\%. Finally, analysis of naturally contaminated maize samples resulted in a good agreement between CL-LFIA and validated confirmatory HPLC-UV and commercial ELISA kit, obtaining recoveries in the range 89-120\%. The proposed CL-LFIA protocol is 20 rapid, inexpensive, easy-to-use, and fit for purpose of rapid screening of mycotoxins in maize flour.

\section{Introduction}

The development of rapid and portable analytical devices for onsite screening applications is one of the most active trends in the field of agrofood analysis, since contamination with toxic 25 substances (e.g., natural toxins, pesticides, veterinary drug residues, environmental pollutants) or microorganisms pose severe safety issues, as well as great economic concern. Immunochromatographic assays (also named lateral-flow immunoassays, LFIA) have shown to be particularly 30 advantageous for such applications, since they provide rapid, simple, specific analyses with no instrumental requirement. Following their success in diagnostics, applications in agrofood screening is now an emerging field. In order to foster the use of LFIA methods as screening tools for food safety, two promising 35 fields of research have been recently identified, namely the development of multiplex assays and their combination with portable recording devices1. Such features will enhance the competing ability of these portable tests with laboratory-based screening methods, directly providing on-site quantitative 40 information on a number of analytes in a given sample. This will enable accurate screening of a large number of samples directly where they are obtained and significant savings in terms of time and costs, since only the actual suspicious samples will be transported to the analytical laboratory for confirmatory analyses. Aflatoxins and Fumonisins are secondary metabolites produced by Aspergillus and Fusarium respectively, growing on agricultural commodities in the field or after harvest2. Since mycotoxins represent one of the most important threat for cereal safety3, exhibiting acute toxic, carcinogenic, mutagenic, 50 teratogenic, immunotoxic and estrogenic effects in man and animals4,5, the European Commission (EC) has established maximum residue limits (MRLs) in cereals and cerealbased foods and feeds: 4000 (g kg-1 for type B-fumonisin and 4 (g kg-1 for the sum of Aflatoxins B1 (AfB1), B2, G1 and G2 as well as 2 (g kg-1 for AfB1655 . 
A wide number of LFIA methods have been developed for mycotoxin detection in feed and food1,7-9. We recently reported a chemiluminescence (CL) LFIA-based biosensor for simple, rapid and ultrasensitive on-site quantification of type B-fumonisins in maize flour down to $25 \mu \mathrm{g} \mathrm{kg}-160$, which has been successfully applied to both standard and real samples10. It is well known that enzymecatalyzed CL detection provides high detectability, rapidity, specificity and wide linear range in immunoassays 11,12, especially in miniaturized formats 13 . This approach has been 65 recently extended to LFIA methods, converting them from qualitative methods (when conventional colloidal gold labelling is employed) to highly sensitive and quantitative assays 14-16. There is a growing demand for multiplex screening assays to replace single-analyte ones, since several mycotoxins may coexist in a single product and yield to synergistic toxic effects2,770. Despite immunochromatography technology potentially offers easy implementation of multi-residue analysis and obvious economic encouragement for this approach, few multiplex LFIA assays have been described in the literature up to now8,17-21, and none of them exploited the advantages of CL detection. Herein we report the development of a biosensor for the multiplex detection of type-B fumonisins and AfB1 in maize 10 samples. The biosensor is based on a portable ultrasensitive CCD-based "contact" imaging device coupled with a CL-LFIA strip, on which two competitive immunoassays are simultaneously performed. Aflatoxin B1 conjugated with bovine serum albumin (AfB1-BSA) and Fumonisin B1 (FmB1)-BSA 15 conjugate were immobilized in different positions along the strip. Upon sample application, type-B fumonisins and AfB1 in the sample competed with immobilized analogues for their specific antifumonisin or anti-aflatoxin antibodies added to the sample. Signal detection was performed by CL contact imaging upon 20 addition of a secondary horseradish peroxidase (HRP)-labelled secondary antibody and the suitable enzyme CL substrate. With this format, a quantitative and objective measurement of target analytes below EU regulatory levels was performed, thus enabling rapid and reliable identification of those samples requiring confirmatory analysis.

\section{Materials and methods Reagents}

FmB1, AfB1, Aflatoxin B2, Aflatoxin G1, Aflatoxin G2, Ochratoxin A, Deoxynivalenol, and Zearalenone (Oekanal 30 certified solutions), BSA, Tween-20 and HRP-labeled goat antirabbit immunoglobulin were purchased from Sigma Aldrich (St.Louis, MO, USA). Ultrapure water was produced by a Millipore Milli Q system (Millipore, Bedford, MA). The Supersignal ELISA Femto CL substrate for HRP was bought from Thermo Fisher Scientific Inc. (Rockford, IL). FmB1 and AfB1 powder were purchased from Fermentek (Jerusalem, Israel). The goat anti-rabbit antibody was purchased from AbCam (Cambridge, UK). Rabbit anti-FmB1 antibodies and rabbit anti-AfB1 antibodies were kindly supplied by Generon srl (Modena, Italy). The other reagents were of analytical grade and were employed as received. Phosphate buffered saline (PBS) was prepared as follows: 10 mmol L-1 Na2HPO4, 2 mmol L-1 5 KH2PO4, 137 mmol L-1 NaCl, 2.7 mmol L-1 KCl, pH 7.4. Assay strips for LFIA were prepared by immobilizing on nitrocellulose membranes, from bottom to top of the strip, the FmB1-BSA conjugate, the AfB1-BSA conjugate, and the goat anti-rabbit 10 antibody to form the two test lines (T-lines) and the control line (C-line), respectively, keeping a distance of 4 $\mathrm{mm}$ between the lines (Fig. 1, Panel C). The membranes were then assembled with a sample and an adsorbent pad and cut into sections. Details are available as Supplementary Material.

\section{Preparation of mycotoxin-BSA conjugates}

The FmB1-BSA conjugate was synthesized according to Christensen et al.22, with slight modifications. Details are available as Electronic Supplementary Information. The AfB1-oxime hapten (AfB1-CMO), synthesized according to Kolosova et al.2320, was employed for the preparation of the AfB1-BSA conjugate by the N-hydroxysuccinimide ester method as follow: $5.7 \mathrm{mg}$ of AfB1-CMO were reacted overnight at room temperature with $5 \mathrm{mg}$ of BSA dissolved in $0.15 \mathrm{M}$ sodium bicarbonate 
pH 8.3 and the pure conjugate was obtained from 25 gel-filtration, as described above. AfB1-BSA concentration was determined through Brilliant Blue Comassie method. Conjugates were supplemented with $0.1 \%$ sodium azide and stored refrigerated.

\section{Instrumentation}

The biosensor, shown in Fig. 1 (Panel B), was assembled employing a previously described CCDbased contact imaging configuration10,24. In particular, the LFIA strip was placed directly in contact with the thermoelectrically-cooled CCD sensor through a round fiber optic taper. A mask was used to ensure reproducible strip positioning. This assembly was enclosed in a dark box to provide shielding from ambient light. During the acquisition the CCD sensor temperature was kept at $-10 \_$C.

\section{Assay procedure}

The nitrocellulose strip was placed horizontally on the larger fiber optic taper surface, then the LFIA assay was started by depositing on the bottom of the strip a volume of 100 / $\mathrm{L}$ of solution, containing $40\lceil\mathrm{~L}$ of PBS with $3 \%$ BSA (w/v) and $0.1 \%$ Tween $20(\mathrm{v} / \mathrm{v}), 5$ (L of HRP-labeled goat anti-rabbit antibody diluted 1:500 (v/v) in PBS, 5 (L of rabbit anti-FmB1 and anti-AfB1 antibody, each diluted 1:500 (v/v) in PBS, and 50 ( $\mathrm{L}$ of maize sample extract (or blank maize sample extract for the blank, or FmB1 and AfB1 standard solutions prepared in blank maize sample extract to produce calibration curves). Upon complete migration of the solution (10 $\mathrm{min})$, the strip was washed by flowing 100 ( $\mathrm{L}$ of PBS for $10 \mathrm{~min}$. Then 70 (L of CL substrate was added at the bottom of the strip and let flow through the membrane $(4 \mathrm{~min})$, which was kept at $25^{\circ} \mathrm{C}$. The $\mathrm{CL}$ signal was acquired with the contact CCDbased imaging device (5-s acquisition time). Total analysis time was about $30 \mathrm{~min}$. The scheme of the multiplex CL-LFIA assay on the nitrocellulose strip is showed in Fig. 1 (Panel A). To obtain quantitative information, the mean photon emission was measured in the areas corresponding to $\mathrm{C}$-line and T-lines of the LFIA strip and each was subtracted of the mean background signal measured in two adjacent areas below and above the line. The $\mathrm{T}$-line/C-line ratio was calculated for each analyte and then converted into B/B0 ratio by dividing it for the T-line/C-line ratio measured in the absence of the target analyte (B0, i.e., maximum T-line/C-line value). Calibration curves were obtained by plotting $\mathrm{B} / \mathrm{B} 0$ values against the log of analyte concentration and fitting the experimental data with a fourparameter logistic equation. Linearization of the calibration curve was obtained through the logit-log transformation, by plotting the logit of the $\mathrm{B} / \mathrm{B} 0$ ratio (as a percentage) against the log of analyte concentration. The best data fit was obtained by linear regression of the standard points.

\section{Analysis of maize samples}

Maize flour samples were obtained directly from producers or mills. Fumonisin content was determined by HPLC-UV as previously described2575. AfB1 contamination was assessed by a commercial ELISA kit (EuroClone SpA, Milano, Italy). Maize flour samples were subjected to a preanalytical extraction procedure previously described10. Briefly, $1 \mathrm{~g}$ of maize flour was suspended in 10

$\mathrm{mL}$ of PBS buffer, hand-shaken for $3 \mathrm{~min}$ at RT and let settle for $5 \mathrm{~min}$. Then, a 100- $(\mathrm{L}$ aliquot of the supernatant was heated for $3 \mathrm{~min}$ at $100{ }^{\circ} \mathrm{C}$ to inactivate endogenous maize peroxidase, then cooled to room temperature and subjected to analysis by LFIA. Heating was performed on a indium-tin oxide (ITO)-coated glass (SPI Supplies/Structure Probe Inc., West Chester, PA) employing a Frame-Seal slide chamber (Bio-Rad Laboratories, Hercules, CA) to contain the sample and avoid evaporation. To obtain the analyte concentration value for each sample, its B/B0 value was calculated as described above and interpolated on a stored calibration curve.

\section{Results and discussion}

A duplex indirect competitive CL-LFIA was developed by depositing on a test strip AfB1-BSA and FmB1-BSA on T-lines and goat anti-rabbit antibody on a unique C-line. With this format, each of the two analytes present in the sample competes with its corresponding immobilized hapten for binding the 
specific anti-AfB1 or anti-FmB1 rabbit antibodies, which are in turn detected by employing HRPconjugated anti-rabbit antibody and CL detection. Anti-rabbit antibodies immobilized on the Cline allow confirmation of correct test development by capturing any rabbit-antibody-based signal reagent (i.e. the presence of the signal indicated the correct migration of the reactants along the strip). Moreover, normalizing the signal of $\mathrm{T}$-lines with respect to that of C-line allows correction of environmental and matrix factors that might affect the intensity of CL signals on the strip (i.e. changes in room temperature or the presence of HRP inhibitors in the sample), thus providing a strip-to-strip normalization factor. Indeed, as previously reported25, it is expected that the C-line intensity is fairly independent from analytes concentration. Signals were detected employing an ultrasensitive cooled CCD sensor employed in a "contact imaging" approach, as previously described. As shown in Figure 1C, 4-mm distance between adjacent lines was sufficient to prevent interference in signal detection. According to the competitive immunoassay principle, the light emission intensity of the T-lines gradually decreased up to their complete disappearance as the concentration of the respective analyte in the sample increased. The $5 \mathrm{C}$-line intensity was employed as a normalization value, to increase assay reproducibty and compensate for test strip to test strip variability.

\section{Optimization of experimental parameters}

Assay parameters (concentration of immunoreagents and selection of the saturation agent) were optimised to generate assays with limits of detection (LOD) and dynamic ranges useful for detecting AfB1 and FmB1 in maize samples below regulatory limits. Furthermore, the methods were optimized considering that, in order to detect simultaneously both analytes, the three lines on a strip should provide similar signal intensity. This ensures the possibility to use a single integration time for the simultaneous measurement of the CL signals on a strip, preventing cross-talk phenomena. The concentrations of primary and secondary antibodies yielding the highest detectability for FmB1 and AfB1 were evaluated. The optimal concentration of anti-fumonisin (1:500 v/v), antiaflatoxin (1:500 $\mathrm{v} / \mathrm{v})$ and HRP-labelled anti-rabbit $(1: 500 \mathrm{v} / \mathrm{v})$ were selected as the dilution that provides the highest detectability and the best compromise between the effects described above. Data referring to the optimization of the immunoreagents dilution are available as Electronic Supplementary Information. Furthermore, different protocols were assayed for saturating the nitrocellulose membrane, thus reducing nonspecific adsorption of the immunoreagents and increasing the signal intensity of the lines, as previously shown25. In particular, different saturation agents (BSA, non fat dry milk, soybean milk) and additives in the running buffer were tested. The best performance was obtained by incubating the nitrocellulose strips with $1 \%$ BSA (w/v) in PBS buffer for $5 \mathrm{~min}$ at room temperature and by adding $3 \%$ BSA (w/v) and $0.1 \%$ Tween $20(\mathrm{v} / \mathrm{v})$ in the running buffer. Finally, the absence of cross reactivity (CR) between the two analytes was checked by running each primary antibody in the absence of analytes in solution. As shown in Fig. 2, no CR between anti-fumonisin antibody and AfB1 (left panel), nor between anti-aflatoxin and FmB1 (right panel) was observed, as confirmed by the absence of the upper and lower T-lines, respectively.

\section{Specificity of the immunoreagents}

The specificity of the immunoassays was determined towards structurally related and unrelated mycotoxins by measuring the 55 IC50 value for each compound of interest, i.e., the concentration of tested compound providing a response at the $50 \%$ bound (midrange of the curve). The CR values were calculated as the ratio of the IC50 value for the analyte over the IC50 value of the tested interfering compound. The anti-aflatoxin antibody showed 60 low CR with AfG2 and AfB2 (below 2\%) and higher for AfG1 (38\%). The anti-fumonisin antibody showed about $100 \% \mathrm{CR}$ with FmB2, thus showing its ability to detect type-B fumonisins present in the sample, as the sum of FmB1 and FmB2. Both antibodies showed very low CR towards Zearalenone, Deoxynilvalenol, and Ochratoxin A, as shown in Table 1. 
Table 1 Cross reactivity values measured for the anti-fumonisin and antiaflatoxin antibodies.

Anti-fumonisin

antibody

Anti-aflatoxin

antibody

Fumonisin B1 100\%<0.02\%

Fumonisin B2 97\%<0.02\%

Aflatoxin B1 $<0.02 \% 100 \%$

Aflatoxin B2 $<0.02 \% 2 \%$

Aflatoxin G1 $<0.02 \% 2 \%$

Aflatoxin G2 $<0.02 \% 38 \%$

Zearalenone $<0.02 \% 0.2 \%$

Deoxynilvalenol $<0.02 \% 0.8 \%$

Ochratoxin A $<0.02 \% 2 \%$

The characteristics of the employed antibodies make the multiplex assay particular relevant for regulatory purposes. Indeed, EU regulations define limit values for fumonisin as the sum of FmB1 and FmB26. In addition, while limit values for aflatoxins are reported as the sum of AfB1, AfB2, AfG1, and AfG2, it is important to note that the ability to singularly detectthe concentration of AfB1 is particular relevant, being this mycotoxin listed as a group 1 carcinogen by the International Agency for Research on Cancer4. Finally, negligible CR of the antibodies towards structurally unrelated mycotoxins considerably reduces the risk for false positive results.

\section{Calibration curves}

Calibration curves were generated by using standard solutions produced in blank maize flour sample extracts in the range between $0.2-1500\lceil\mathrm{~g} \mathrm{~L}-1$ and $0.06-50\lceil\mathrm{~g} \mathrm{~L}-1$ of FmB1 and AfB1, respectively. Calibration curves were produced first by working in single assay; in particular both 5 primary antibodies were added in all the strips, while the two analytes were assayed separately. As shown in Fig. 3, being a competitive type format, the decrease of T-line/C-line ratio was directly proportional to the amount of the analyte in the sample (the T-lines completely disappeared at the highest FmB1 and AfB1 concentration).

Table 2 Comparison between limit of detection found for Aflatoxin B1 and Fumonisin $\mathrm{B} 1$ in previous work.

Fumonisin B1 Aflatoxin B1

This work $0.6 \mu \mathrm{g} \mathrm{L}-1$

$(6 \mu \mathrm{g} \mathrm{kg}-1)(\mathrm{a})$

$0.15 \mu \mathrm{g} \mathrm{L}-1$

$(1.5 \mu \mathrm{g} \mathrm{kg}-1)$

Mirasoli et al. 2012 [10] $2.5 \mu \mathrm{g} \mathrm{L-1}$

(25 $\mu \mathrm{g} \mathrm{kg}-1)$

-

Anfossi et al. 2010 [25] $12 \mu \mathrm{g} \mathrm{L-1}$

(120 $\mu \mathrm{g} \mathrm{kg}-1)$

Anfossi et al. 2011 [26] - $0.1 \mu \mathrm{g} \mathrm{L-1}$

(1 $\mu \mathrm{g} \mathrm{kg}-1)$

Molinelli et al. 2009 [27] $199 \mu \mathrm{g} \mathrm{kg-1} \mathrm{(a)}$ 
Wang et al. 2006 [28] $1 \mu \mathrm{g}$ L-1 -

Lattanzio et al. 2012 [19] $3200 \mu \mathrm{g} \mathrm{kg-1} \mathrm{(a)} \mathrm{-}$

Wang et al. 2013 [18] $5.23 \mu \mathrm{g} \mathrm{L-1} \mathrm{-}$

Reveal $®($ Neogen

Corporation) (b)

$1 \mathrm{mg} \mathrm{kg}-120 \mu \mathrm{g} \mathrm{L}-1$

Agrastrip (Romer Labs)

(a)

$0.2 \mathrm{mg} \mathrm{kg}-14 \mu \mathrm{g} \mathrm{L}-1$

Quick Tox

(EnviroLogix) (a)

$3200 \mu \mathrm{g} \mathrm{kg}-120 \mu \mathrm{g} \mathrm{L}-1$

$a$ As the sum of Fumonisin B1 and B2

b Monoplex assay format, offered for a variety of mycotoxins. Limits of

detection are referred to total fumonisins or total aflatoxins concentration.

The limit of detection (LOD) was calculated as the concentration corresponding to the blank T-line/Cline value minus three times the blank standard deviation. The obtained values were $0.6(\mathrm{~g} \mathrm{~L}-1 \mathrm{FmB} 1$ and $0.15 \int \mathrm{g} \mathrm{L}-1 \mathrm{AfB} 1$, corresponding respectively to $6 \int \mathrm{g} \mathrm{kg}-1$ and $1.5(\mathrm{~g} \mathrm{~kg}-125$ in maize flour samples (according to the extraction procedure employed in this work). The dynamic range of the method extended from 0.6 to $1500 \Upsilon \mathrm{g} \mathrm{L}-1$ for $\mathrm{FmB} 1$ and from 0.15 to 50 (g L-1 for AfB1, with a midpoint value at 40 /g L-1 and 0.9 /g L-1, respectively. 30 The LOD values obtained for FmB1 and AfB1 are comparable or lower than the those reported in the literature employing a CLLFIA 10 or colloidal gold based LFIA assays25-28, as well as when compared with commercial assays (Table 2). The obtained results are comparable with those of recently published articles which 35 describe the use of indirect competitive LFIA based on colorimetric detection for a multiple semi-quantitative determination of Fusarium mycotoxins in cereals 19 and for the simultaneous quantification of ZEA and FmB1 in corn and wheat18. The results of repeated calibration curves demonstrated a 40 good reproducibility, the relative standard deviation associated to each point of the calibration curve being $0.5-12 \%$ for FmB1 and 0.5-7\% for AfB1, respectively (3 calibration curves produced in separate runs for each analyte). Finally, calibration curves obtained separately for each analyte (although in the presence of 45 both primary antibodies) were compared with those obtained by working in multiplex format, adding in the same sample known amounts of both FmB1 and AfB1. To evaluate the possibility of accurately detecting each analyte, even when one is present at a higher concentration with respect to the other, multiplex 50 calibration curves were generated either by increasing the amount of both analytes simultaneously, or by increasing the concentration of one analyte while decreasing that of the other.

A representative series of CL images and the obtained calibration curves are 5 shown in Fig. 4. Results show that the simultaneous presence of the two analytes in the sample, even in disproportionate amounts, does not affect the light emission intensity of the other test line. The LOD and the dynamic range obtained in monoplex format 10 were confirmed also by working in multiplex assay, making it possible to detect and quantify the two mycotoxins in a unique analysis. This indicates that multiplex competitive assays on a single test strip can produce quantitative assays without loss in assay detectability as compared with single tests.

\section{Maize flour samples}

To enable multiplex assays in a point-of-use format, a simple and rapid sample preparation procedure, providing good recovery for all the analytes of interest, must be employed. It has been previously 
shown that extraction in aqueous media at close to neutral $\mathrm{pH}$ values provides good recovery for both aflatoxins26 20 and fumonisins 25. Furthermore, the absence of organic solvents in the extraction solution makes the procedure more environmentalfriendly and avoids problems of disruption of antigen-antibody binding. Thus, maize flour samples were subjected to extraction 25 in PBS buffer, followed by rapid extract heating to inactivate peroxidase enzymes naturally present in maize (which was performed employing a portable system suitable for point-of-use applications). The whole analytical procedure, including sample preparation, lasted $30 \mathrm{~min}$. 30 The assay analytical performance was evaluated by analyzing pooled extracts from blank maize flour samples (previously analyzed by ELISA) fortified by adding known amounts of both FmB1 (166, 18 and 2 (g L-1) and AfB1 (1.8, 0.6 and $0.21(\mathrm{~g} \mathrm{~L}-1)$, corresponding approximately to $0.9,0.6$ and $0.3 \mathrm{~B} / \mathrm{B} 0$ values on 35 the respective calibration curve. Recovery ranged from 79 to $119 \%$, while coefficients of variation below $20 \%$ were obtained (Table 3 ).

The performance of the multiplex LFIA method was also evaluated on real samples and results were compared with those 40 obtained by confirmatory analyses, performed by HPLC-UV (FmB1 + FmB2 content) and a commercial ELISA kit (AfB1). Results, reported in Table 3 show a good agreement between CLLFIA and reference methods for both mycotoxins in all samples, with recovery values ranging from 89 to $120 \%$ and coefficient of 45 variation below $20 \%$. A picture of the membranes used for the analyses of the maize samples is reported in Fig. 5.

Table 3 Results obtained in the analysis of maize flour samples by CL-LFIA biosensor. (A) Fortified samples were produced by adding known amounts

of fumonisin B1 and aflatoxin B1 to a blank maize sample extract. (B) Maize flour samples, previously analyzed by HPLC-UV and ELISA, were

subjected to extraction and analyzed by CL-LFIA biosensor. CL-LFIA data are expressed as mean and CV\% of three independent measurements.

(A) Maize flour extracts fortified with fumonisin B1 and aflatoxin B1 (B) Maize flour samples

Expected

concentration

$(\mu \mathrm{g} \mathrm{L}-1)$

CL-LFIA ( $\mu \mathrm{g} \mathrm{L}-1)$ Recovery (\%) Expected

concentration

$(\mu \mathrm{g} \mathrm{L}-1)$

CL-LFIA ( $\mu \mathrm{g} \mathrm{L}-1)$ Recovery $(\%)$

Fumonisin Fumonisin

$166148(\mathrm{CV} \%=5) 89470478(\mathrm{CV} \%=20) 101$

$1814.6(\mathrm{CV} \%=15) 798087(\mathrm{CV} \%=12) 108$

$22.3(\mathrm{CV} \%=13) 1151012(\mathrm{CV} \%=15) 120$

Aflatoxin Aflatoxin

$1.81 .72(\mathrm{CV} \%=19) 920.450 .43(\mathrm{CV} \%=10) 95$

$0.60 .7(\mathrm{CV} \%=12) 1130.570 .58(\mathrm{CV} \%=10) 102$

$0.210 .20(\mathrm{CV} \%=14) 950.500 .44(\mathrm{CV} \%=15) 88$

\section{Conclusions}

The results show that the multiplex biosensor 5 provides reliable, with performances comparable with those obtained employing reference methods. The extraction procedure fulfils the requirements for the development of a method that can be applied on site. The co-extraction of the two mycotoxins, although based 10 on a simple manual extraction with aqueous buffer and rapid heating system employing a portable manifold, shows satisfactory recoveries. The method is rapid (total analysis time 
$30 \mathrm{~min}$ ), simple, cost effective and can be performed in a non-laboratory environment by nonspecialists. 15 The possibility to detect simultaneously two analytes, providing quantitative results within current regulatory limits, reduces the number of analysis and allows to screen on site a large number of samples, thus reducing the costs for transporting and analyzing samples in the laboratory. This allows performing frequent 20 analyses monitoring the entire production chain (e.g., on field, at harvest, during storage and transportation) according with the HACCP procedures. In the future, it will be possible to further increase the number of analytes by adding other test lines, provided the compatibility with the antibody employed in the 25 immunoassay. Ongoing work is devoted to the development of a ready-to-use cartridge containing the LFIA strip and all the reagents necessary per a complete analysis, in order to facilitate point-of-use applications.

\section{Notes and references}

a Department of Chemistry “G. Ciamician”, University of Bologna, via Selmi 2, 40126 Bologna, Italy

b Department of Chemistry, University of Turin, Via P. Giuria, 5, 10125

35 Torino, Italy

c National Institute for Biostructures and Biosystems (INBB), Viale

Medaglie d'Oro 305, 00136 Rome, Italy

$\dagger$ Electronic Supplementary Information (ESI) available: [details of any

40 supplementary information available should be included here]. See

DOI: $10.1039 / \mathrm{b} 000000 \mathrm{x} /$

\$ Footnotes should appear here. These might include comments relevant to but not central to the matter under discussion, limited experimental and spectral data, and crystallographic data.

45

1. B.B. Dzantiev, N.A. Byzova, A.E. Urusov and A.V. Zherdev, Trend Anal Chem, 2014, 55, 81-93.

2. R. Krska, P. Schubert-Ullrich, A. Molinelli, M. Sulyok, S. MacDonald and C. Crews, Food Addit Contam, 2008, 25, 152-163.

50 3. Codex Alimentarius, (1991). Codex standard for durum wheat semolina and durum wheat flour 178-1991 (Rev. 1-1995). Rome:

FAO/WHO.

4. International Agency for Research on Cancer, IARC, 1993, pp.301-366 IARC (1993) International Agency for Research in Cancer, Lyon, 55 France. IARC 57:427-794

5. M. Edite Bezerra da Rocha, F.daC.O. Freire, F. Erlan Feitosa Maia, M. Izabel Florindo Guedes and D. Rondina, Food Control, 2014, 36, 159-165.

6. Commission Regulation EC No 1881/2006 (2006) Off J Eur Union 60 364:5-24; Commission regulation (EC) No 1126/2007 (2007) Off J Eur Union 255:14-17.

7. I.Y. Goryacheva, S. De Saeger, S.A. Eremin and C. Van Peteghem, Food Addit Contam, 2007, 24, 1169-1183.

8. L. Anfossi, C. Baggiani, C. Giovannoli, G. D’Arco and G. Giraudi, 65 Anal Bioanal Chem, 2013, 405, 467-480.

9. R. Krska and A. Molinelli, Anal Bioanal Chem, 2009, 393, 67-71. 10. M. Mirasoli, A. Buragina, L.S. Dolci, P. Simoni, L. Anfossi, G.

Giraudi and A. Roda, Biosens. Bioelectron, 2012, 32, 283- 287. 
11. A. Roda, M. Mirasoli, M. Guardigli, E. Michelini, P. Simoni and M. Magliulo, Anal Bioanal Chem, 2006, 384, 1269-1275.

12. M. Magliulo, M. Mirasoli, P. Simoni, R. Lelli, 5 O. Portanti and A.

Roda, J Agric Food Chem, 2005, 53, 3300-3305.

13. M. Mirasoli, M. Guardigli, E. Michelini and A. Roda, J Pharm

Biomed Anal, 2014, 87, 36-52.

14. I.H. Cho, E.H. Paek, Y.K. Kim, J.H. Kim and S.H. Paek, Anal. Chim. 10 Acta, 2009, 632, 247-255

15. M. Mirasoli, A. Buragina, L.S. Dolci, M. Guardigli, P. Simoni, A. Montoya, E. Maiolini, S. Girotti and A. Roda, Anal Chim Acta, 2012,

721, 167- 172.

16. H-A. Joung, Y.K. Oh and M-G. Kim, Biosens Bioelectron, 2014, 53, $15330-335$.

17. B. Ngom, Y. Guo, X. Wang and D. Bi, Anal Bioanal Chem, 2010, 397, 1113-1135.

18. Y. Wang, Y. Yan, W. Ji, H. Wang, S. Li, Q. Zou and J. Sun, J Agric

Food Chem, 2013, 61, 5031-5036.

20 19. V.M.T. Lattanzio, N. Nivarlet, V. Lippolis, S. Della Gatta, A.C. Huet, P. Delahaut, B. Granier and A. Visconti, Anal. Chim. Acta, 2012,

718, 99-108.

20. P. Li, Z. Zhang, Q. Zhang, N. Zhang, W. Zhang, X. Ding and R. Li, Electrophoresis, 2012, 33, 2253-2265.

25 21. E. Njumbe Ediage, J.D. Di Mavungu, I.Y. Goryacheva, C. Van Peteghem and S. De Saeger, Anal Bioanal Chem, 2012, 403, 265278.

22. H.R. Christensen, F.Y. Yu and F.S. Chu, J Agric Food Chem, 2000, 48, 1977-84.

30 23. A.Y. Kolosova, W.B. Shim, Z.Y. Yang, S.A. Eremin, D.H. Chung, Anal Bioanal Chem, 2006, 384, 286-294.

24. A. Roda, M. Mirasoli, L.S. Dolci, A. Buragina, F. Bonvicini, P.

Simoni and M. Guardigli, Anal Chem, 2011, 83, 3178-3185.

25. L. Anfossi, M. Calderara, C. Baggiani, C. Giovannoli, E. Arletti and 35 G. Giraudi, Anal Chim Acta, 2010, 682, 104-109.

26. L. Anfossi, G. D’Arco, M. Calderara, C. Baggiani, C. Giovannoli and G. Giraudi, Food Addit Contam Part A Chem Anal Control Expo Risk Assess, 2011, 28, 226-234.

27. A. Molinelli, K. Grossalber and R. Krska, Anal Bioanal Chem, 2009, 40 395, 1309-1316.

28. S. Wang, Y. Quan, N. Lee and I.R. Kennedy, J Agric Food Chem, 2006, 54, 2491-2495.

45 